\title{
Therapeutic Role of Garlic Extracts in Aging and Age- Related Diseases
}

\author{
EunJin Bang ${ }^{1}$, Hee Jin Jung ${ }^{1}$, Dae Hyun $\mathrm{Kim}^{1}{ }^{1}$, Su Gyeong Ha ${ }^{1}$, Takako Yokozawa ${ }^{2}$ and Hae Young \\ Chung*1 \\ ${ }^{1}$ Department of Pharmacy, College of Pharmacy, Pusan National University, Busan 46241, Republic of Korea \\ ${ }^{2}$ Graduate School of Science and Engineering for Research, University of Toyama, Toyama 930-8555, Japan
}

*Corresponding author: Hae Young Chung, Department of Pharmacy, College of Pharmacy, Pusan National University, Busan 46241, Republic of Korea

ARTICLE INFO

Received: 炭 June 05, 2019

Published: 幽 June 11, 2019

Citation: EunJin Bang, Hee Jin Jung, Dae Hyun Kim, Su Gyeong Ha, Takako Yokozawa, Hae Young Chung. Therapeutic Role of Garlic Extracts in Aging and Age-Related Diseases. Biomed J Sci \& Tech Res 18(5)-2019. BJSTR. MS.ID.003203.

Abbreviations: GE: Garlic Extracts; ROS: Reactive Oxygen Species; NF: Nuclear Factor; SAC: S-Allylcysteine; CAT: Catalase; SOD: Superoxide Dismutase; GPx: Glutathione Peroxidase; NO: Nitric Oxide; RA: Rheumatoid Arthritis; CIA: Collagen-Induced Arthritis; MDA: Malondialdehyde; CRP: C-Reactive Protein; PWV: Pulse Wave Velocity; CAC: Coronary Artery Calcium; AD: Alzheimer's Disease

\section{ABSTRACT}

As aging population is increasing, there is growing needs and interest in healthy aging and delaying onset and progression of age-related diseases such as Alzheimer's disease, atherosclerosis, arthritis, diabetes mellitus, obesity and cancer. Aging is defined as the accumulation of detrimental changes observed at both molecular and cellular levels, which ultimately leads to a functional decline or physiological changes in tissues and organs. In particular, dietary nutritional intervention provides various health benefits without serious side effects in delaying onset of age-related diseases. Garlic has been consumed for hundreds of years as a natural health medication. The active ingredient of garlic contains organosulfur compounds, which are well known to exert antioxidant, anti-inflammatory, cardioprotective, antimicrobial and anticancer effects through modulatory mechanism on oxidation and inflammatory response. As oxidative stress and chronic systemic inflammation are some of the major risk factors underlying aging and age-related diseases, anti-oxidative and anti-inflammatory intervention could promote prevention of age-related disease onset and progression. Therefore, garlic is assuring research field in regard to the therapeutic potential as monotherapeutic or adjuvant agent to conventional therapeutics for aging and age-related diseases. In this mini review, we discuss the potential therapeutic role of garlic extract to promote healthy aging and prevent age-related diseases.

Keywords: Garlic Extracts; Age-Related Diseases; Oxidative; Inflammation; Diabetes Mellitus; Atherosclerosis; Arthritis

\section{Introduction}

Inrecentyears,increasinginteresthasbeenshownintheresearch field of aging and age-related diseases. Aging is a multifactorial process that is accompanied by changes in molecular and cellular signaling pathway and morphology. Cells undergoing chronological aging are associated with an accumulation of intracellular reactive oxygen species (ROS), increased activity of the transcription factor nuclear factor (NF)- $\mathrm{B}$ and subsequent hyper induction of inflammatory target genes, cytokines and chemokines such as TNF- $\alpha$, IL-1 $\beta$, IL-6, IL-8 and MCP-1 [1,2]. This intracellular oxidative stress and chronic inflammation are considered as major risk factors underlying mechanism of aging and age-related diseases. Aging is one of the critical risk factors associated with ultimate functional decline oforgans and tissues and compromise physiological homeostasis. As aging declines ability to restore homeostasis, it leads to dysfunction of organs and causes pathogenesis of agerelated disease such as atherosclerosis, arthritis, obesity, type 2 diabetes, Alzheimer's disease and cancer [3,4]. In order to prevent early onset of age-related diseases, demands for healthy aging and therapeutic intervention are increasing.

The dietary active compounds could be obtained from functional foods, herbs, and natural products such as ginseng, nuts, grains and polyphenols, which have been consumed for healthy aging and stimulation of rejuvenation as their healthy aging effect is highly supported by epidemiological studies [5,6]. Among dietary interventions, garlic (Allium sativum) has long been historically documented for ancient medicinal uses associated with aging [7]. Aged garlic extraction and isolation (GE) is one of important main bioactive preparation. The aging process of aged garlic extracts for 
20 months, the S-allylcysteine (SAC) is increased, which is known for its strong antioxidant effect $[8,9]$. As there varies different forms of active compounds in garlic products, effects depend on its main isolated ingredients. Here, the health benefits such as anti-oxidative, anti-inflammatory, anti-microbial, anti-cancer, and cardioprotective effects will be discussed based on previous in vivo and in vitro studies.

\section{Molecular Mechanism of Garlic Extracts for Anti-Aging Effects}

\section{Garlic and Antioxidant Effects}

Underlying risk factor of age-related diseases is accumulation of ROS and oxidative stress. As anti-oxidative dietary compounds, aged garlic extracts co-supplemented with vitamin B, folic acid, L-arginine demonstrated protective effects against free radical accumulation. For example, alliin scavenged free radicals and allicin suppressed the superoxide formation and both allyl cysteine and allyl disulfide successfully scavenged hydroxyl radicals [10]. In diabetic rat model with exacerbated oxidative stress, garlic (250 $\mathrm{mg} / \mathrm{kg}$ ) administration upregulated transcription of antioxidant genes, catalase (CAT), superoxide dismutase (SOD), and glutathione peroxidase (GPx) in the blood and heart tissue [11] and ameliorated mitochondria dysfunction and increased SIRT-3, MnSOD, which correlates with reduction of ROS levels in heart [12]. In addition, levels of NO and TNF- $\alpha$ were decreased close to the normal levels in kidney of diabetic rat model [13]. In addition, RoS production and malondialdehyde levels of skin and serum were notably suppressed by garlic administration and prevented skin photoaging accelerated by UV irradiation [14]. The administration of SAC induced activation of $\mathrm{Nrf}_{2}$, a transcription factor that activates transcription of antioxidant genes in the striatum and hippocampus of brain [9].

\section{Garlic and Anti-inflammatory Effects}

Systemic, low-grade chronic inflammation is one of the major persistent causes of aging and age-related diseases. Three active garlic compounds, caffeic acid, SAC, and uracil exhibited antiinflammatory effects by downregulating pro-inflammatory COX2 and iNOS genes in UVB-irradiated hairless mouse model [15] In cell studies, HaCaT cells pre-treated with garlic before UVB irradiation significantly reversed UVB-induced upregulation of pro-inflammatory cytokine secretion such as IL-6 and IL-1 $\beta$ similar to that of UVB-untreated normal conditions [16]. The antiinflammatory activities of aged garlic extract notably suppressed the NO generation and TNF- $\alpha$ and prostaglandin- $\mathrm{E}^{2}$ dose-dependently in lipopolysaccharide (LPS)-treated macrophage cells. At molecular levels, inhibitory effects of MAPK and NF-kB were demonstrated $[17,18]$. In X-ray irradiated mouse model, allylmethylsulfide from garlic inactivated NF-kB transcription factor and suppressed its pro-inflammatory target genes iNOS, COX-2, and VCAM-1 through inhibition of MAPK pathway [19].

\section{Therapeutic Effects of Garlic Extracts in Age-Related Diseases}

\section{Rheumatoid Arthritis}

Aging is an important risk factor for chronic inflammatory disease such as rheumatoid arthritis (RA). Diallyl trisulfide isolated from garlic bulb exerted modulatory effects in the rheumatoid arthritis pathogenesis. In collagen-induced arthritis (CIA) mouse model and human fibroblast-like synoviocytes from RA patients, diallyl trisulfide alleviated levels of pro-inflammatory cytokines via suppression of NF- $\kappa \mathrm{B}$ and Wnt signaling pathway and regulated the immune function restoration [20].

\section{Garlic in Diabetes Mellitus}

Aging process is accompanied by impairment of energy homeostasis and carbohydrate metabolism. Hyperglycemia is considered to be due to deficiency of insulin secretion and growing insulin resistance with aging. As hyperglycemia is accompanied by increased intracellular levels of free radicals, numbers of studies found that garlic administration leads to significant reductions in fasting glucose level based on meta-analysis of randomized controlled trial studies [21]. In diabetic nephropathy rat model, garlic treated diabetic model had lower levels of malondialdehyde (MDA), nitric oxide (NO) levels when compared to diabetic rats in kidney [13].

\section{Garlic in Atherosclerosis}

Atherosclerosis is associated with aging and its pathogenesis involves endothelial cell proliferation, DNA damage and vascular oxidative stress, and chronic inflammation. Modulatory effects of garlic have been suggested by invitro and invivo studies although reduction of atherosclerosis-associated hyperlipidemia and hypercholesterolemia is relatively modest in comparison to therapeutic effects of commercially prescribed medications. Based on literature review examining modulatory action of garlic supplements on vascular disease parameters and biomarkers, garlic showed beneficial effects on reduction of cardiovascular risk factors such as hypertension and total cholesterol and atherosclerosis surrogate markers, C-reactive protein (CRP), pulse wave velocity (PWV), and coronary artery calcium (CAC) [22].

\section{Garlic in Alzheimer's Disease}

Alzheimer's disease (AD) is a progressive neurodegenerative disease and is primarily characterized by dementia. The currently approved treatment strategies and medications for Alzheimer' disease are limited and treatment has not been effectively curable. Previous in vivo study reported that decreased tau phosphorylation, which is related to pathogenesis of AD is achieved by treatment of aged garlic extracts by suppression of GSK-3 $\beta$ activity [23] Furthermore, allicin from garlic extract is reported to prevent and ameliorate cognitive impairment of learning and memory 
capabilities based on AD mouse model. Allicin also reduced the expression level of $A \beta$ in extracellular neuritic plaque composed of $A \beta$. In addition, allicin improved mitochondria function and decreased oxidative status through JNK/c-JUN signaling pathway in AD mouse model [24].

\section{Conclusion}

With increasing aging population, preventive intervention for aging and age-related disease has been gathering growing interest. The garlic has been favorably known for its antioxidant, anti-inflammatory, antimicrobial, and anticancer effects in agerelated disease conditions. As oxidation and systemic low-grade chronic inflammation is major underlying mechanism of aging and age-related disease, garlic may be an effective dietary intervention decreasing risk and delaying aging and onset of age-related diseases. In order to understand and confirm therapeutic relation between garlic extracts and age-related diseases, well-organized, long-term human clinical trials on dietary supplementary intake would be necessary and need to further examine molecular mechanism of active compounds of garlic extracts.

\section{Acknowledgement}

This work was supported by a Cooperative Research Program for Agriculture Science \& Technology Development (Project No. PJ006522132013) of Rural Development Administration, Republic of Korea. In addition, we thank Aging Tissue Bank for providing research information. This work was supported by a National Research Foundation (NRF) grant funded by the Korean government (MSIP) (Grant No. 2015M3A9B8029074).

\section{Conflict of Interest}

The author declares no conflict of interest.

\section{References}

1. Chung HY, Cesari M, Anton S, Marzetti E, Giovannini S, et al. (2009) Molecular inflammation: underpinnings of aging and age-related diseases. Ageing Res Rev 8(1): 18-30.

2. Giorgi C, Marchi S, Simoes ICM, Ren Z, Morciano G, et al. (2018) Mitochondria and Reactive Oxygen Species in Aging and Age-Related Diseases. Int Rev Cell Mol Biol 340: 209-344.

3. Hartl FU (2016) Cellular Homeostasis and Aging. Annu Rev Biochem 85: $1-4$.

4. Shlisky J, Bloom DE, Beaudreault AR, Tucker KL, Keller HH, et al. (2017) Nutritional Considerations for Healthy Aging and Reduction in AgeRelated Chronic Disease. Adv Nutr 8(1): 17-26.

5. Yang Y, Ren C, Zhang Y, Wu X (2017) Ginseng: An Nonnegligible Natural Remedy for Healthy Aging. Aging Dis 8(6): 708-720.

6. Agatonovic Kustrin S, Kustrin E, Morton DW (2019) Essential oils and functional herbs for healthy aging. Neural Regen Res 14(13): 441-445.

7. Rahman K (2003) Garlic and aging: new insights into an old remedy. Ageing Res Rev 2(1): 39-56.
8. Elosta A, Slevin M, Rahman K, Ahmed N (2017) Aged garlic has more potent antiglycation and antioxidant properties compared to fresh garlic extract in vitro. Sci Rep 7: 39613.

9. Franco Enzastiga U, Santana Martinez RA, Silva Islas CA, Barrera Oviedo D, Chanez Cardenas ME, et al. (2017) Chronic Administration of S-Allylcysteine Activates Nrf2 Factor and Enhances the Activity of Antioxidant Enzymes in the Striatum, Frontal Cortex and Hippocampus. Neurochem Res 42(11): 3041-3051.

10. Chung LY (2006) The antioxidant properties of garlic compounds: allyl cysteine, alliin, allicin, and allyl disulfide. J Med Food 9(2): 205-213.

11. Naderi R, Mohaddes G, Mohammadi M, Alihemmati A, Badalzadeh R, et al. (2015) Preventive effects of garlic (Allium sativum) on oxidative stress and histopathology of cardiac tissue in streptozotocin-induced diabetic rats. Acta Physiol Hung 102(4): 380-390.

12. Sultana MR, Bagul PK, Katare PB, Anwar Mohammed S, Padiya R, et al. (2016) Garlic activates SIRT-3 to prevent cardiac oxidative stress and mitochondrial dysfunction in diabetes. Life Sci 164: 42-51.

13. Nasiri A, Ziamajidi N, Abbasalipourkabir R, Goodarzi MT, Saidijam M, et al. (2017) Beneficial Effect of Aqueous Garlic Extract on Inflammation and Oxidative Stress Status in the Kidneys of Type 1 Diabetic Rats. Indian J Clin Biochem 32(3): 329-336.

14. Kim HK (2016) Garlic Supplementation Ameliorates UV-Induced Photoaging in Hairless Mice by Regulating Antioxidative Activity and MMPs Expression. Molecules 21(1): 70.

15. Kim SR, Jung YR, An HJ, Jang EJ, Choi YJ, et al. (2013) Anti-wrinkle and anti-inflammatory effects of active garlic components and the inhibition of MMPs via NF-kappaB signaling. PLoS One 8(9): e73877.

16. Kim HK (2016) Protective Effect of Garlic on Cellular Senescence in UVBExposed HaCaT Human Keratinocytes. Nutrients 8(8).

17. Kim MJ, Yoo YC, Kim HJ, Shin SK, Sohn EJ, et al. (2014) aged black garlic exerts anti-inflammatory effects by decreasing no and proinflammatory cytokine production with less cytoxicity in LPS-stimulated raw 264.7 macrophages and LPS-induced septicemia mice. J Med Food 17(10): 1057-1063.

18. Jeong YY, Ryu JH, Shin JH. Kang MJ, Kang JR, et al. (2016) Comparison of Anti-Oxidant and Anti-Inflammatory Effects between Fresh and Aged Black Garlic Extracts. Molecules 21(4): 430.

19. Lee EK, Chung SW, Kim JY, Kim JM, Heo HS, et al. (2009) Allylmethylsulfide Down-Regulates X-Ray Irradiation-Induced Nuclear Factor-kappaB Signaling in C57/BL6 Mouse Kidney. J Med Food 12(3): 542-551.

20. Liang JJ, Li HR, Chen Y, Zhang C, Chen DG, et al. (2019) Diallyl Trisulfide can induce fibroblast-like synovial apoptosis and has a therapeutic effect on collagen-induced arthritis in mice via blocking NF-kappaB and Wnt pathways. Int Immunopharmacol 71: 132-138

21. Wang J, Zhang X, Lan H, Wang W (2017) Effect of garlic supplement in the management of type 2 diabetes mellitus (T2DM): a meta-analysis of randomized controlled trials. Food Nutr Res 61(1): 1377571.

22. Varshney R, Budoff MJ (2016) Garlic and Heart Disease. J Nutr 146(2): 416s-421s.

23. Chauhan NB and Sandoval J (2007) Amelioration of early cognitive deficits by aged garlic extract in Alzheimer's transgenic mice. Phytother Res 21(7): 629-640.

24. Zhang Y, Xu L, Ding M, Su G, Zhao Y (2019) Anti-obesity effect of garlic oil on obese rats via Shenque point administration. J Ethnopharmacol 231: 486-493. 


\section{ISSN: 2574-1241}

DOI: 10.26717/BJSTR.2019.18.003203

Hae Young Chung. Biomed J Sci \& Tech Res

(C) (P) This work is licensed under Creative

Submission Link: https://biomedres.us/submit-manuscript.php

$\begin{array}{ll}\text { BIOMEDICAL } & \text { Assets of Publishing with us } \\ \text { RESEARCHES } & \text { - Global archiving of articles } \\ & \text { - Immediate, unrestricted online access } \\ & \text { - Rigorous Peer Review Process } \\ \end{array}$

